\title{
Designing Matter: An Introduction to Architectural Design For First Year Graduate Students
}

\section{ROGER HUBELI}

Syracuse University
BRITT EVERSOLE

Syracuse University

Animals, vegetables, minerals and pixels partake of the world of architecture. The architect's imaginary includes the simplest found substances-copper, aluminum, wood, water, air, light, bytes[1] , plastics, concrete, dirt and clay. Inspired by their physical, chemical, digital and biological possibilities, design could be understood as the aesthetic organization of animate matter into form and composition. [Celant, Arte Povera] The initial act of design is a comprehension and exhibition of a given substance's behavior, from growth to decay, from chemical reaction to movement, deflection and failure.

A process of design that starts with matter "moves away from emphasizing the subject, representation, and interpretation, which characterized [the] 20th century [...] toward focusing instead on the object, material process and expression." [Skylar Tibbits, Active Matter] Focusing on material experimentation provides insight in how designing through innovation - at the scale of matter - can make architecture a site of production "where theory - in the form of a contemplative observation - and experiment [are] inseparably interconnected." [Moravánszky, Ákos, Metamorphism: material change in architecture] For theory and experimentation of a material to co-exist, design requires one to start at the level of matter that is not yet materialized.

Therefore, in order to innovate, one would question the status quo and design at the scale of matter that is not yet a materialized product. As Sheila Kennedy states in Material Presence, "It may seem counter-intuitive for a critical practice of material research to examine the material predicaments inherent in the culture of production as a source of inspiration. But it is precisely here that the greatest challenges to the imagination lie."

With this the students set out their architectural career with a notion that architecture is an inherent part of the material environment that surrounds them. Providing the students with a notion that architectural design requires engaging the social and environmental conditions that are so pertinent for our built environment. 

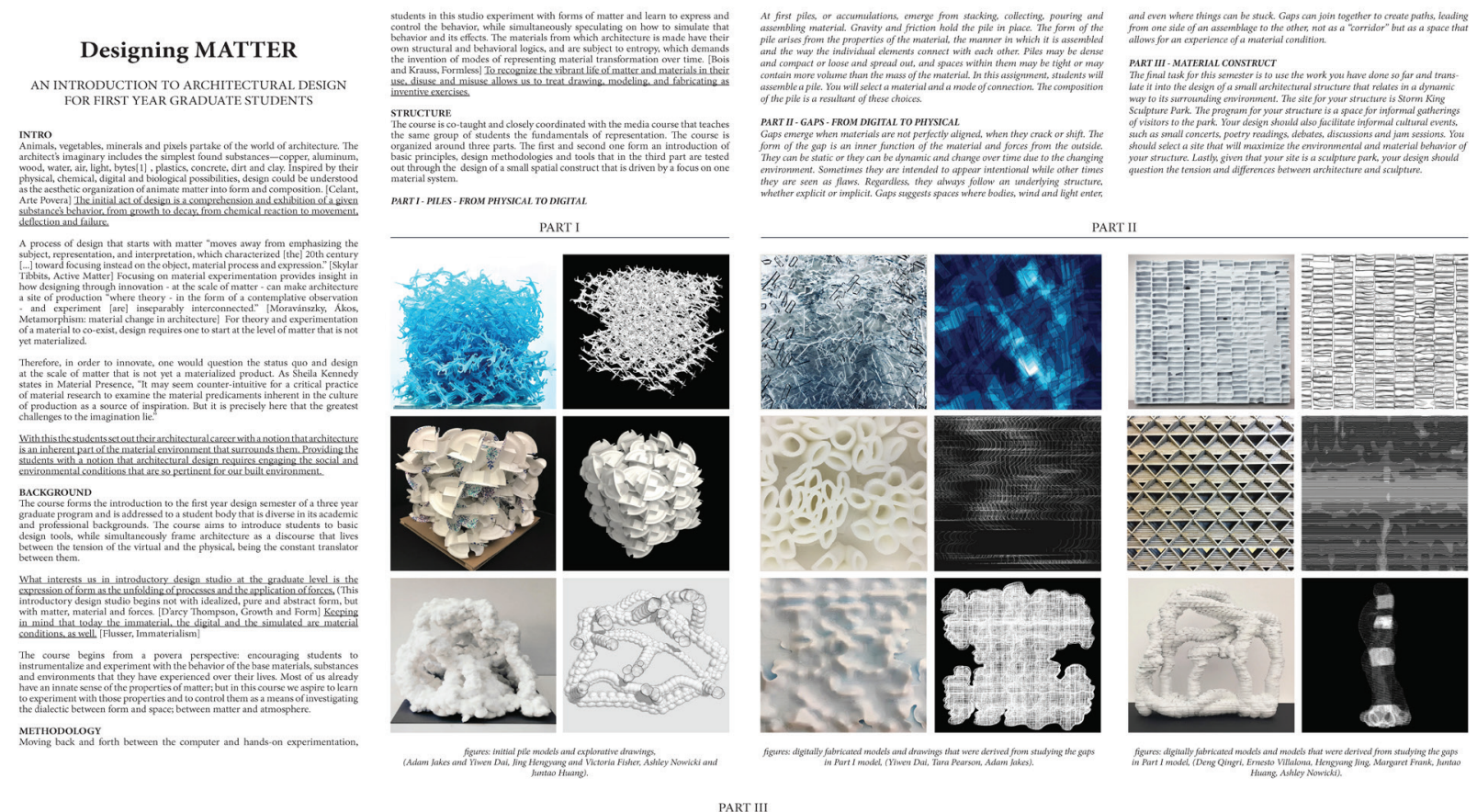

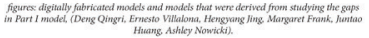
PART III

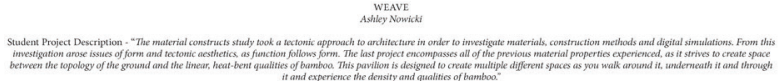

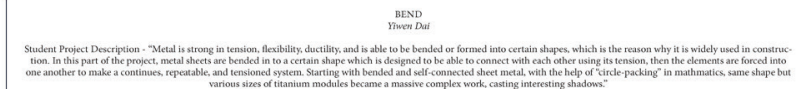
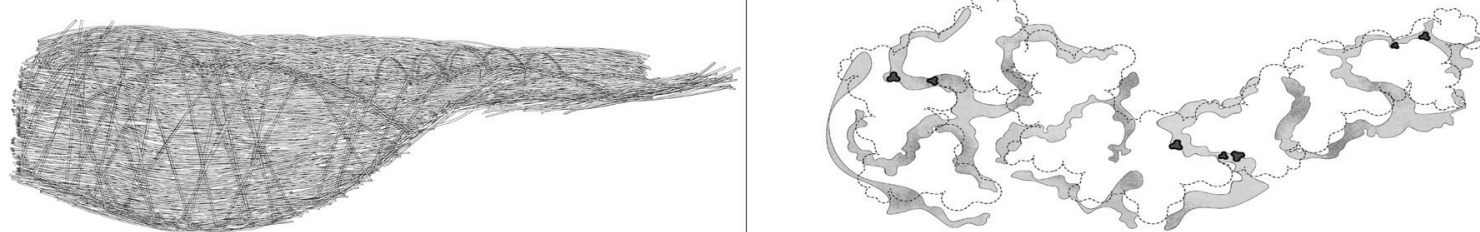

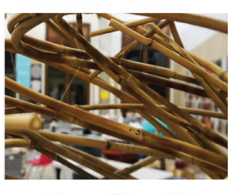

( Pि

1(1)

(c) 2 (a)
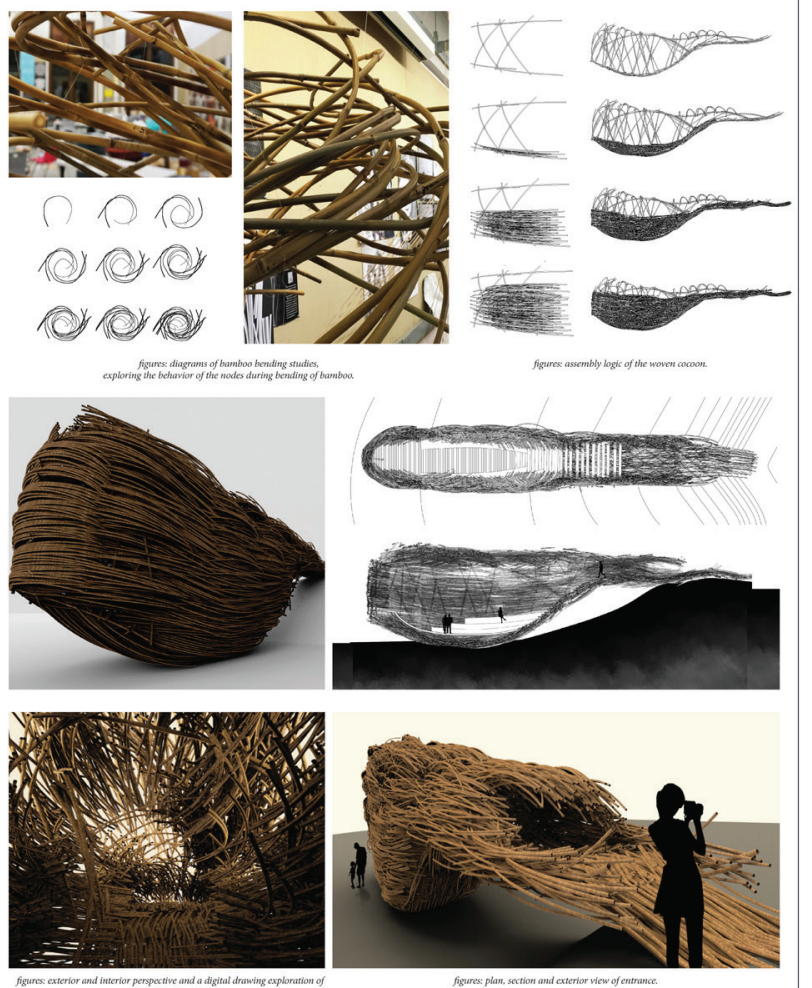